\title{
CHALLENGES IN FLYING QUADROTOR UNMANNED AERIAL VEHICLE FOR 3D INDOOR RECONSTRUCTION
}

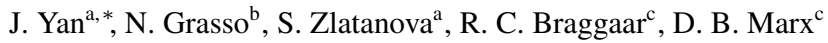 \\ a 3D Geoinformation, Delft University of Technology, 2628 BL Delft, the Netherlands - \\ (j.yan-3, s.zlatanova)@tudelft.nl \\ ${ }^{\mathrm{b}}$ Politecnico di Torino, DIATI - Department of Environmental, Land and Infrastructure Engineering, C.so Duca degli \\ Abruzzi 24, 10129, Torino, Italy, nives.grasso@ polito.it \\ ${ }^{c}$ Geomatics for the Built Environment, Delft University of Technology, Julianalaan 134, 2628 BL Delft, the Netherlands, \\ r.c.braggaar@tudelft.nl,d.b.marx@student.tudelft.nl
}

\section{Commission VI, WG VI/4}

KEY WORDS: Challenges, Indoor, 3D Modelling, Quadrotor Unmanned Aerial Vehicle (QUAV)

\begin{abstract}
:
Three-dimensional modelling plays a vital role in indoor 3D tracking, navigation, guidance and emergency evacuation. Reconstruction of indoor 3D models is still problematic, in part, because indoor spaces provide challenges less-documented than their outdoor counterparts. Challenges include obstacles curtailing image and point cloud capture, restricted accessibility and a wide array of indoor objects, each with unique semantics. Reconstruction of indoor environments can be achieved through a photogrammetric approach, e.g. by using image frames, aligned using recurring corresponding image points (CIP) to build coloured point clouds. Our experiments were conducted by flying a QUAV in three indoor environments and later reconstructing 3D models which were analysed under different conditions. Point clouds and meshes were created using Agisoft PhotoScan Professional. We concentrated on flight paths from two vantage points: 1) safety and security while flying indoors and 2) data collection needed for reconstruction of 3D models. We surmised that the main challenges in providing safe flight paths are related to the physical configuration of indoor environments, privacy issues, the presence of people and light conditions. We observed that the quality of recorded video used for 3D reconstruction has a high dependency on surface materials, wall textures and object types being reconstructed. Our results show that 3D indoor reconstruction predicated on video capture using a QUAV is indeed feasible, but close attention should be paid to flight paths and conditions ultimately influencing the quality of 3D models. Moreover, it should be decided in advance which objects need to be reconstructed, e.g. bare rooms or detailed furniture.
\end{abstract}

\section{INTRODUCTION}

Three-dimensional modeling is critical for a variety of indoor applications including 3D tracking (Sebe et al., 2004), 3D navigation ( $\mathrm{Li}$ and $\mathrm{He}, 2008$ ), 3D guidance (Hagedorn et al., 2009), emergency evacuation (Lee and Zlatanova, 2008) and robotic applications (Hornung et al., 2013). However, the reconstruction of indoor 3D models is still problematic seeing as image and point cloud capture in indoor spaces presents a different set of challenges than outdoor spaces. Examples of these challenges include physical constraints hindering movement (Li, 2008)(Yang and Worboys, 2011a), restricted accessibility, scale/dimensions of indoor spaces (Yang and Worboys, 2011b) and recognition of types of objects with different semantics (Stoffel et al., 2007). These challenges hamper attempts at automation of reconstruction of indoor 3D models (Zlatanova et al., 2013). Nevertheless, these issues can be partially rectified by leveraging methodologies pertaining to photogrammetry or laser scanning; tools and methods originally employed for outdoor 3D modeling. There are two main data sources for outdoor spatial reconstruction: images and point clouds. Images and point clouds can be obtained using photogrammetric techniques from long distances (i.e. remote sensing or airborne photogrammetry), or short distances (i.e. close-range photogrammetry). Using hand-held laser scanners to collect indoor point clouds will not provide color and texture simultaneously. Therefore, close-range photogrammetry is

\footnotetext{
${ }^{*}$ Corresponding author
}

suitable for collection of data at indoor scales, since this technique uses overlapping, multi-perspective aerial image sequences (or in this case, video frames) for object positioning purposes. Close-range photogrammetry is well-suited for 3D reconstruction (Remondino et al., 2014), since it allows for rendering of object geometries and textures as well. In recent years, unmanned aerial vehicles (UAV) have become commonplace for aerial imagery collection, as they are able to fly through limited-access zones and can thereby be utilized as platforms for mapping and surveillance (Nex and Remondino, 2014) (Teuliere et al., 2010). Moreover, many software programs have been developed to reconstruct three-dimensional models with photos or videos. Examples include Agisoft Photoscan (Manual, 2014), Pix4D (Strecha, 2011), 3DF Zephyr (Nikolov and Madsen, 2016), Global Mapper (LLC, 2009), and EasyUAV (Koska, 2011). Although low resolution, motion blur and redundancy of frames lessen the utility of multi-perspective image sequences for video-based reconstruction (Alsadik et al., 2015), these tools can make 3D reconstruction easier, faster and less expensive. Currently, few researchers address indoor 3D model reconstruction based on this method, in which videos are obtained by flying a UAV. This paper investigates challenges in designing flight paths for video capture using a Quadrotor Unmanned Aerial Vehicle (QUAV), the Parrot Bebop 2. In order to do this, we conducted various experiments with the Parrot Bebop 2 in three unique indoor environments. Later, we reconstructed 3D models and analyzed their quality under a variety of different conditions. These $3 \mathrm{D}$ reconstruction processes 
were performed using Agisoft PhotoScan Professional. In our research, we concentrate on flight path design from two primary vantage points: 1) safety and security while flying indoors and 2) data collection needed for reconstruction of indoor 3D models. The content of this paper is organized as follows: after discussing challenges of flying QUAV for 3D indoor reconstruction in Section 2, we present our methodology and flight path designs in Section 3. We discuss our case study and conduct of experiments complete with results in Section 4. Finally, our conclusion and future work are presented in Section 5.

\section{CHALLENGES IN FLYING QUAV FOR 3D INDOOR RECONSTRUCTION}

Indoor space is defined as space within one or more buildings consisting of architectural components such as entrances, corridors, rooms, doors and stairs (Lee et al., 2014) (Li, 2008). Indoor space has features which differ considerably from outdoor space (Yan et al., 2016). Of these, this paper addresses four main features of indoor space having inevitable effects on the application of flying QUAV for 3D model reconstruction; specifically: constraints, accessibility, objects and semantics.

\subsection{Constraints}

The main difference between flying QUAV in indoor spaces versus outdoor spaces are physical constraints. The nature of indoor space is determined by constraints presented by architectural components such as doors, corridors, floors, walls and stairs (Yang and Worboys, 2011b). In most cases, no constraints are present in Euclidean outdoor space, especially for UAV which can fly almost anywhere to collect data not prohibited by legal restrictions and regulations. In indoor space, however, these constraints forbid QUAV and other moving things (e.g. people, robots) from traversing walls from one room or space to another without using doors or windows.

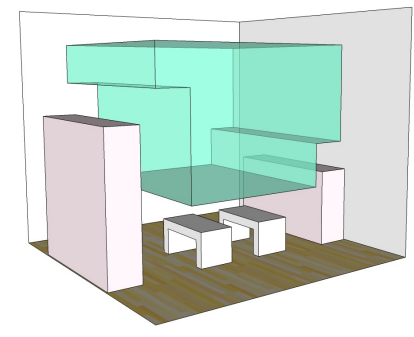

Figure 1. Free space for UAV in indoor environments.

Quite commonly, indoor spaces are shaped like boxes, having uniform length, width and height. Prior to QUAV flights, we outlined free spaces for its movement using optimal distances, (i.e. buffered zones from obstacles for safety), which took into account length, width and height of indoor spaces we modelled. Since obstacles, such as walls, furniture, pillars and objects hanging from the ceilings can easily cause a QUAV to crash, so we only flied the UAV in free space (the green portion of Figure 1).

\section{Safety Issues}

Indoor spaces are enclosed by walls, columns and furniture. As such, there are many obstacles leading to safety issues for QUAV. Some spaces are too low, while others are too high, too small or too dangerous to fly a drone. Seeing as this is the case, we planned collision-free flight paths based on four factors: the size of the QUAV, the field of view (FOV) of the camera, $\theta$ (as seen in Figure $2 b$, the goal of data collection and the dimensions of indoor environments. As shown in Figure 2, the QUAV should have a predefined safety distance, $D$, to walls and other obstacles (as well as people). We tried to ensure flight paths where walls and floors could be viewed simultaneously by the camera embedded in the QUAV, taking into account $D$ and $\theta$. For some experiments, FOV was modified between flight paths to observe changes in level of detail given to objects in our 3D models. Furthermore, we did not attempt to fly the Parrot Bebop 2 within safety distances of furniture (such as tables, see Figure 3), because it would likely have led to crashes.

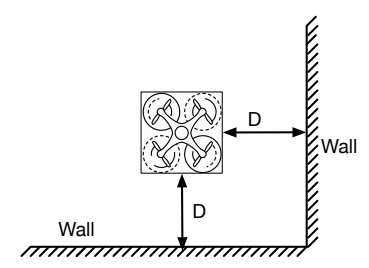

(a)

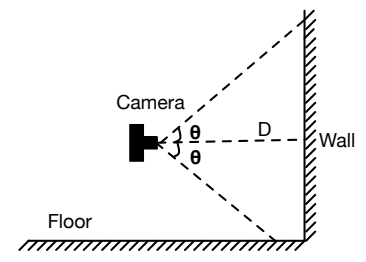

(b)
Figure 2. (a) safety distances for data acquisition as seen from above the QUAV, (b) the FOV of the camera carried by the QUAV from a side-looking vantage point.

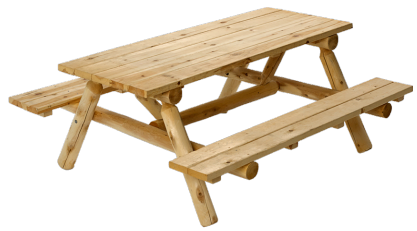

Figure 3. Table.

\subsection{Accessibility}

Space can be characterized by one of three categories based on accessibility: Private, Communal, or Open Access (Paasch et al., 2015). Private spaces are only legally accessible to a private party. Such a party can be one individual, a married couple, group of people, corporate body or non-profit organization. Communal space is subject to a right of common existing within a community where each member has the right to use its holdings independently. Open Access spaces have rights assigned to everyone; no one can be excluded. As is known to all, the majority of outdoor spaces are Communal or Open Access, but indoor space is generally Private. In other words, indoor space has accessibility restrictions. Typical accessibility restrictions are present in places like offices which are only open to certain staff members during certain hours in certain rooms. Likewise, almost all rooms are forbidden for non-staff members in places like hospitals. Furthermore, people cannot enter private homes without the consent of the tenant. As such, UAV face the issue of accessibility in indoor space like people do, but often to a far greater extent.

\section{Accessibility Issues}

An example of a place inaccessible to UAV are art museums where it is forbidden to take pictures. Therefore, we cannot fly UAV in museums and buildings with similar rules outlawing image capture. In addition to bans of image and video capture, people in indoors spaces are often perturbed by the volume and gust generated by UAV overhead. Some people perceive UAV to 
be dangerous. Many will not consent to being photographed or recorded. In areas, such as this, UAV can never be flown. Another example of a place that is forbidden for UAV flight is hospitals. Buildings such as this ban UAV flight, because they rely upon a quiet and safe environment for medical treatment at all hours of day and night. Most private buildings, likewise forbid UAV flight, for reasons specified or implied, unless permission is sought and granted by owners of these buildings.

\subsection{Objects}

In indoor space, most objects are artificial, small and numerous. Objects fall into one of five categories according to CityGML (Gröger et al., 2012): Doors, Windows, Walls, Building Installations and Building Furniture. However, for our 3D reconstruction purposes, we classify indoor objects into the following categories: architectural components, regularly-shaped furniture and irregularly-shaped furniture. Architectural components are necessary parts of buildings such as walls, floors, ceilings, doors and windows. Regularly-shaped furniture is furniture which has at least one surface that is regularly-shaped and larger than $0.5 \mathrm{~m} 2$, such as box-like fixtures (e.g. cabinets, lockers, sofas and tables). Irregularly-shaped furniture consists of furniture and other objects with small surfaces such as chairs, books, phones, power lines and clothes.

\section{Issues with Objects}

Issues with objects often arise from texturing. Objects can be highly reflective, transparent or not textured at all. Data collection is quite difficult when capturing blank walls, glass products and furniture. To make indoor spaces brighter, many walls are purposefully left blank and white. There are often decorations and other small objects attached to walls, but many of them are at a low position near the ground. Therefore, during certain experiments, it was quite difficult to find suitable places for CIPs placement necessary for photo alignment to process data for blank walls devoid of distinctive features. Another issue is presented by glass objects like windows and other transparent furniture. Windows can produce serious reflections off of UAV cameras and can also bring about large deformations of point clouds and meshes on account of improper alignment caused by the capture of outdoor objects. Another problem is furniture. While QUAV have with high manoeuvrability, they still cannot safely traverse most furniture, necessitating flight paths not breaching safety distances mentioned in section 2.2.

\subsection{Semantics}

Semantics are important for indoor 3D models, because they assign meaning to objects based on their attributes (Horna et al., 2007). Without semantics, objects within images would not be discernible from one another. Semantics also provide meaningful signs and symbols which allow people or vehicles to better choose how to move via semantic-based navigation. For instance, semantic-based navigation, can be understood through spoken directions. A theoretical flight path could be described in semantics as: "flying straight along the Urbanism corridor then turning right, where there is a large room called BG.West.030. Once inside this room, fly left until you 've arrived at the office of the 3D Geoinformation Research Group." In this example, the Urbanism corridor, room, BG.West.030, office and 3D Geoinformation Research Group are semantics necessary to comprehend the trajectory of a theoretical flight path.

\section{Semantic Issues}

Objects in indoor spaces have their own semantics which can be classified for a variety of applications. For example, people wanting to find a printer or coffee machine can use models constructed from data captured using a QUAV. We have to know and classify which object in this model is a printer and which is a coffee machine. It's very easy for people to interpret this information from photos, but a tough job for computers to do, since there are many different objects in indoor space. Even the computer cannot distinguish objects by their shapes, seeing as many objects in indoor space have similar shapes. Moreover, in logistical terms concerning reconstructing 3D models, the sofa is part of the wall without semanitcs, since it is positioned against the wall itself. Semantics cannot be obtained and reconstructed from videos/photos directly; a major flaw for our methodology employing close-range photogrammetry based on QUAV flights for indoor 3D model reconstruction.

\subsection{Other Issues}

\section{Issues with Light}

For the purposes of our experiments, data acquisition is the process of recording videos or capturing photos via the camera embedded in the QUAV. The level of light has a big influence on the quality of photos and videos, seeing as poor lighting may cause underexposed photos, further affecting the quality of final $3 \mathrm{D}$ models. However, natural light in indoor space is not always enough. Therefore, we supplemented natural light with artificial light in order to guarantee that the camera received enough light to obtain clear data.

\section{Issues with Noise}

Noise in indoor 3D models is also an issue, although we can try to improve models by establishing goals for how they should look. Sometimes, however, we cannot remove all of the noise and are left with uneeded points in our model. For instance, when we needed an indoor 3D model without furniture, attention was paid to walls, floors and ceilings. For the sake of indoor 3D model reconstruction, people who cross flight paths during video capture are unnecessary and unwanted because they cause noise distorting the objects behind them.

\section{Issues with Occlusion}

Occlusion was another impactful issue in our experiments. Image frames with objects such as light fixtures hanging from ceilings and pillars blocking objects behind them are highly detrimental to CIPs based alignment in Photoscan. The algorithm Photoscan has in place for marker detection is not sufficient for placement of CIPs where they cannot be seen in select frames as a result of occlusion. Nonetheless alignment in Photoscan demands CIPs in every frame be marked, even if their location is occluded by objects much closer to the QUAV camera. This leads to poor results in resulting meshes and point clouds where occlusion makes alignment difficult to attain.

\section{METHODOLOGY}

\subsection{Overview}

Our workflow for flying QUAV for 3D indoor reconstruction, includes three stages (nine steps), as seen above in Figure 4. During the first stage, we set goals for reconstruction and planned flight paths based on the shape of the space modelled. During the second stage, the Parrot Bebop 2 recorded video for data collection and Matlab was used to extract image frames from our videos. The third stage was comprised of five data processes which were conducted using PhotoScan: adding CIPs, photo alignment, building dense point clouds, building meshes 
and building textures which resulted in textured indoor 3D models.

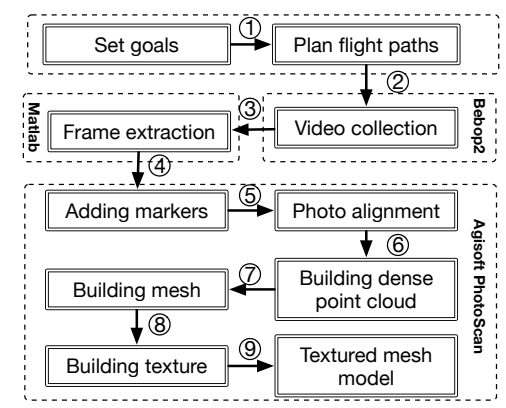

Figure 4. Our workflow for flying QUAV for 3D indoor reconstruction.

\subsection{Flight Path Design}

The quality of the videos/images obtained directly determines the quality of the final three-dimensional model. In order to guarantee the quality of photos, we strove to fly the QUAV in suitable ways as dictated by indoor spatial dimensions. We payed close attention to the most important condition for photos, the overlap requirement; $60 \%$ of side overlap and $80 \%$ of forward-facing overlap per consecutive frame. We designed flight paths based on two principles: 1, safety and security flying QUAV in indoor spaces and 2, data collection needed for the reconstruction of indoor 3D models. There are three suggestions for optimal image and video capture for the QUAV camera (Manual, 2014). For data acquisition of a faade, the QUAV should keep its on-board camera facing the faade while the QUAV itself moves horizontally, parallel to the faade, at an even height halfway between floor and ceiling, rather than remaining at one location and rotating the on-board camera. For data collection of an entire indoor space, the QUAV should circle the space in an ovular pattern. During this time the QUAV camera should always be pointed inwards toward the direct opposite ends of the room, rather than picking spots near corners and rotating $360^{\circ}$ without changing the way the camera faces. For isolated objects. In this scenario, the sensor should record video or take pictures around the object at many different locations, not only front, back, left and right, but also many angular positions.

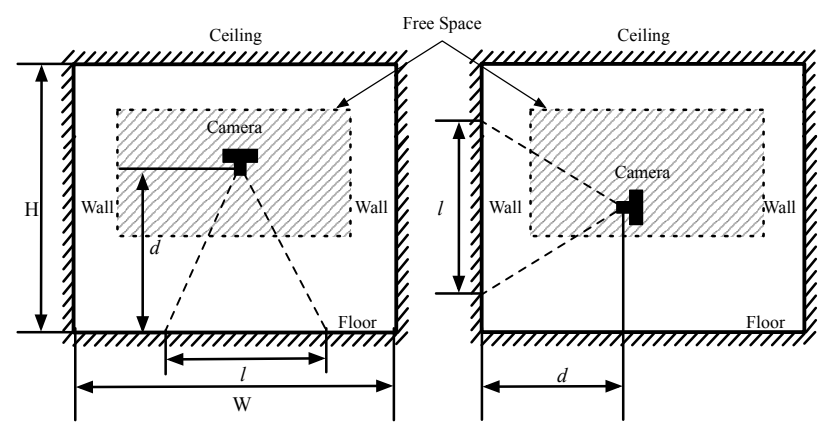

Figure 5. Camera positioning, free space and the FOV of the camera.

We classified indoor spaces into three categories: rooms, corridors and stairs. Based on these categories we designed different flight path strategies incorporating four factors: the size of the
UAV, the FOV of the camera, the goal of data collection and the physical dimensions of data collection environments. To guarantee high quality $3 \mathrm{D}$ models while expending the least possible motion, we designed flight paths for the QUAV based on the data acquisition suggestions. Height and width dimensions of indoor space, $H$ and $W$, the FOV of the camera, $l$, as well as the requirements for side overlap (60\%), together determine the size of free space (shadowed areas in Figure 5).

We designed flight paths based on the relationships between $W$ and $l$, as seen in Equation 1, width and field of view juxtaposed with corresponding flight paths.

$$
\left\{\begin{array}{lc}
W<0.6 l & (e) \operatorname{or}(f) \\
0.6 l \leq W<0.6 l & (a) \operatorname{or}(b) \\
W \geq 0.8 l & (a) \operatorname{or}(b) \operatorname{or}(c) \operatorname{and}(d)
\end{array}\right.
$$

where $\quad W=$ the size of the indoor space

$l=$ the FOV of the cameras

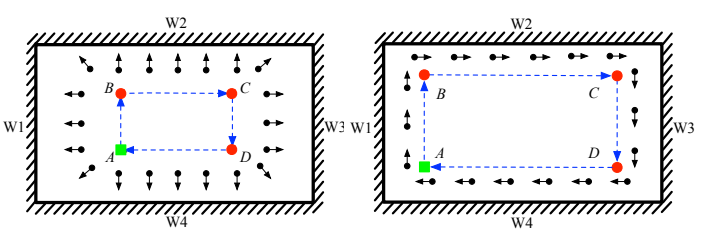

(a)

(b)

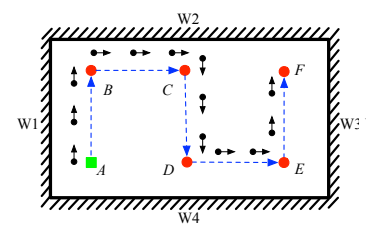

(c)

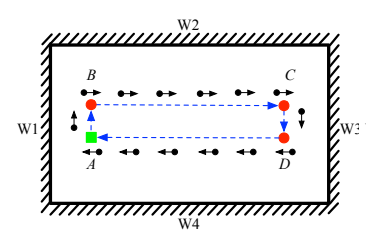

(e)

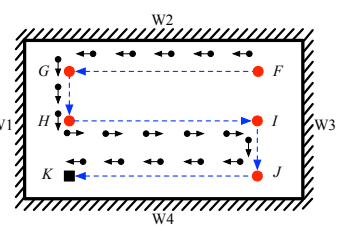

(d)

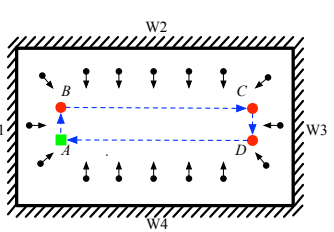

(f)
Figure 6. Flight paths for the QUAV.

Figure 6 illustrates the flight paths for the QUAV. In these flight paths, green and black squares are the starting points and ending points respectively. Red dots are the turning points and the arrow with the black dotted tail denotes the direction of the camera. The thick lines with black slashes comprise the wall and blue dotted arrows are flight paths. For environments where $W$ is larger enough than $l$, (e.g. large rooms or halls) we wanted to procure data for architectural components only. This was done in a manner depicted in Figures 10a and 10b. When we wanted data including furniture, we would follow Figure $6 c$ and $6 d$. For environments where $W$ was less than $0.8 l$, but greater than $0.6 l$, (e.g. long, narrow rooms or corridors), whatever data we wanted to collect was obtained by flying along flight paths depicted in Figures $6 a$ and $6 b$. Otherwise, when $W$ is smaller than $0.6 l$, we 
used flight paths depcited in Figure $6 e$ or $6 f$. These six flight path strategies can also be combined to create new flight paths for differently-shaped spaces. For example QUAV capture of Tshaped rooms, could benift form merging flight path strategies featured in Figure $6 a$ and $6 f$.

\subsection{Data Processing}

Test were performed with the purpose of analyzing the possibility to acquire and use QUAV video capture for indoor 3D model reconstruction. This allowed for evaluation of the effectiveness of our methods and provided a platform to identify procedural weaknesses. Alignment of images acquired by the QUAV camera was made possible by using the Structure from Motion (SfM) approach implemented in Photoscan. Input data required for this tool was only images since it was not necessary to know a priori interior and exterior parameters of these cameras. In the first phase, the image elaboration lead to tie point extraction and photo alignment. This alignment algorithm found matching pixels by creating a sparse 3D point cloud. Once the images are aligned, it is then possible to import the Ground Control Points (GCPs), if available, to perform a bundle block adjustment (Boccardo et al., 2015) and compute the absolute orientation as well as georeferenced the sparse point cloud (Manual, 2014). The next step toward indoor 3D model reconstruction was the generation of dense 3D point clouds. Subsequently, a triangulated mesh was produced and textured using images acquired from QUAV video capture.

\subsection{Parameter Estimations}

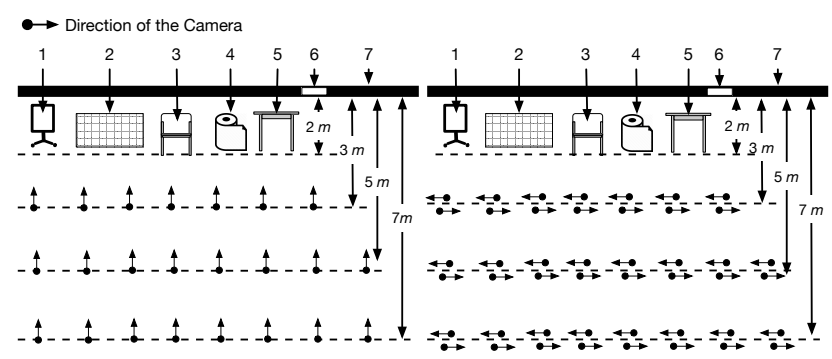

(a)

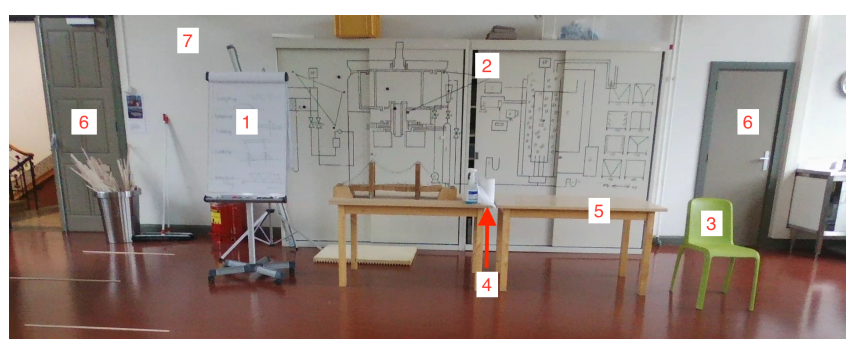

(b)

Figure 7. Forward-facing view of flight paths for parameter estimations.

Objects 1-7, as featured in Figures 7 are as follows: 1. Movable Whiteboard, 2. Locker, 3. Chair, 4. Roll of Paper Towels, 5. Table, 6. Doors and 7. Wall. Figures $7 a$ left and $7 a$ right depict the direction of QUAV flights and camera positions for these particular experiments. Figure $7 b$ is an image of objects as they appeared in the TU Delft Science Centre Workshop 5 environment. In order to conduct parameter estimations for object reconstruction pertaining to flight paths in Section 3.3, we carried out two specific experiments seen in Figure $7 a$. It was our intention to merge key characteristics of previous flight paths in these experiments. In parameter estimation experiments we took all objects in indoor space into consideration including architectural components (e.g. walls, windows and doors), regularly-shaped furniture (e.g. movable whiteboards, lockers and tables) and irregularlyshaped furniture (e.g. rolls of paper, trash cans and chairs).

\section{EXPERIMENTS}

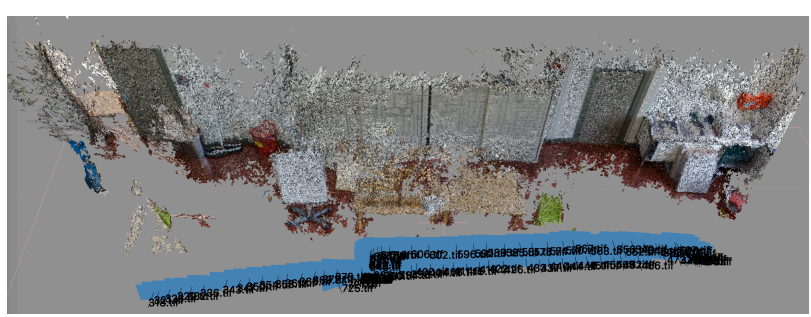

(a)

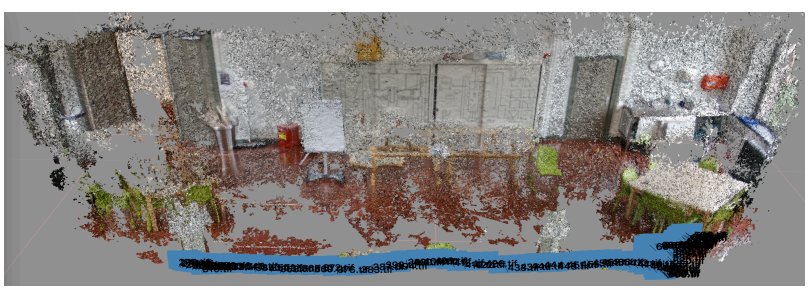

(b)

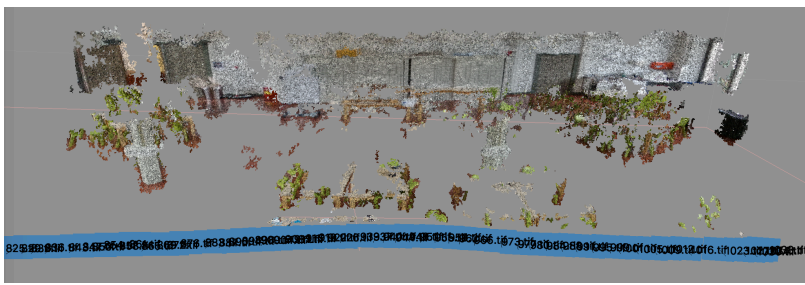

(c)

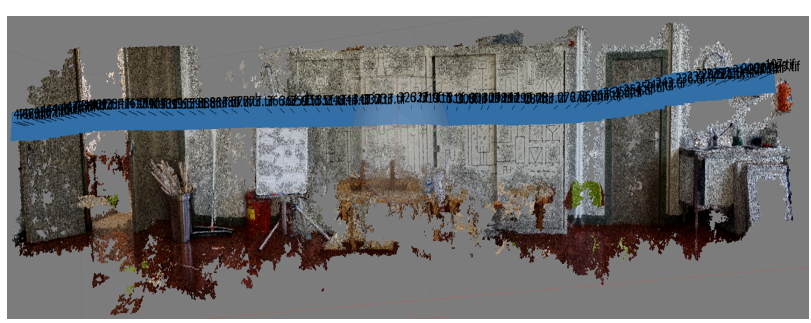

(d)

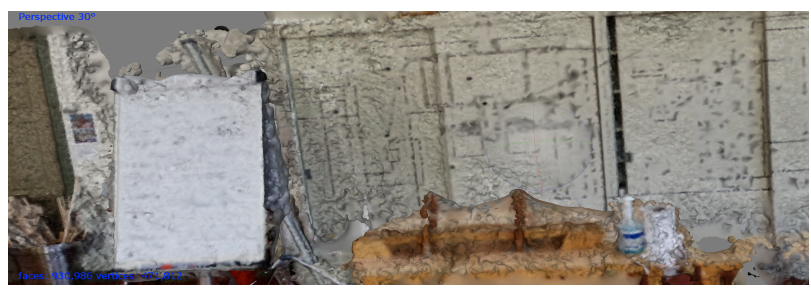

(e)

Figure 8. Dense Point clouds with aligned photos from parameter estimation experiments.

Eight flight paths collected data at distances of three, five and seven meters between the QUAV camera and the wall. We gen- 
erated point clouds of the first three paths, see Figure 8, in which the on-board camera is based on Figure $7 a$. For Figure $8 a$, the distance between the on-board camera and wall was three meters, while the distance between the camera and objects on the table was one meter. For Figure $8 b$, the distance was five meters to the wall and three meters to the objects on the table. For Figure $8 c$, the distance was seven meters from camera to wall and five meters from camera to table. Like Figure $8 a$, Figures $8 d$ and $8 e$ were produced from the flight path one meter from the table and three meters from the wall. However, unlike Figures $8 a, 8 b$, and $8 c$, the dense point cloud pictured in Figure $8 d$ was produced with ultrahigh quality rather than high quality in Photoscan, and the direction of the on-board camera is based on $7 b$. Seeing as Figure $8 d$ depicts successfully rendered irregularly-shaped objects on the table, while Figure $8 a$ has no such clarity, we infer that ultra-high processing quality and the patience that requires is necessary to depict irregularly-shaped objects, even from as close as one meter away. Figure $8 e$ confirms that meshes produced from ultra-high quality dense clouds do indeed depict irregularly-shaped objects with high levels of clarity. Nonetheless, gaps in the ultra-high quality dense point cloud still suggest that occlusion is an issue cannot be circumnavigated by processing power.

The results show that around 1 meter from the camera, furniture is suitable for $3 \mathrm{D}$ model reconstruction. However, the greater the distance between the camera and objects, the less realistic meshes became. Within five meters, wall reconstruction was fair, but around seven meters, the distance became too far to reconstruct any objects, even large, flat surfaces like a wall. In Figure $9 \mathrm{a}$ and $9 \mathrm{~b}$, the door, locker, movable whiteboard, table and washbasin areas are comprised of pockets of dense points. However, due to subpar renderings of irregularly-shaped furniture, increasing distance from camera to object and large swaths of indoor space missing as a result of occlusion from pillars, challenges in indoor $3 \mathrm{D}$ reconstruction will likely persist in similar spaces where data capture is conducted with faulty flight paths.

\subsection{Case Studies}

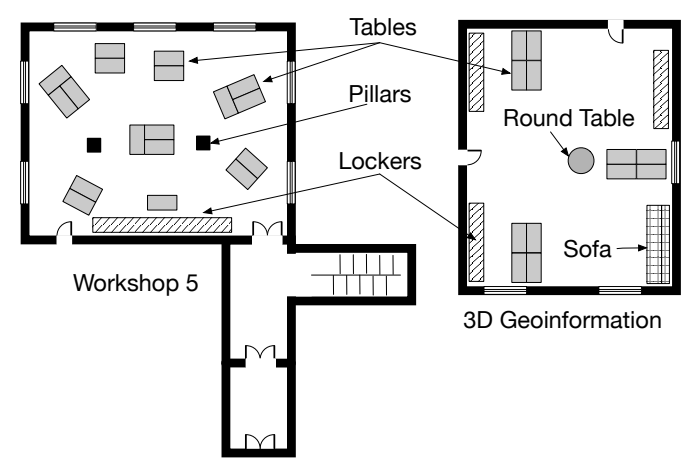

Figure 9. Floor layouts of experiment environments.

Initially, we planned to reconstruct 3D mesh models with architectural components, (e.g. walls, doors, windows, corridors and stairs) and regularly-shaped furniture, (e.g. tables, lockers and sofas). These flight paths were created and flown in places such as a corridor outside Workshop 5 which connects with a stairwell (as seen in Figures 9 ). A series of experiments were also conducted in the office of the 3D Geoinformation Research Group (length: 11 meters, width: 7.1 meters, height: 5 meters) at Delft University of Technology and Workshop 5 (length: 8.5 meters, width:
6 meters, height: 3.5 meters) in the TU Delft Science Centre. With the exception of two central pillars and three low-hanging light fixtures that run the width of Workshop 5, both Workshop 5 and the office of the 3D Geoinformation Research Group are indoor spaces with typical layouts: doors, windows, furniture, floors and walls (as seen in Figure 9). In the office of 3D Geoinformation Research Group, there are three lockers, three sets of tables, two doors, three windows, one round-table and one sofa. While eight sets of tables, two pillars, one locker, two doors and seven windows are present in Workshop 5. It is noteworthy that there are many irregularly-shaped objects which cannot be filtered out directly, such as monitors, keyboards, phones, cups and books. Based on the above parameter estimation experiment, we set the FOV of the camera of the Bebop 2 at $90^{\circ}$. The best data acquisition distance from the on-board camera to objects in this experiment was found to be roughly 1-2 meters. Our QUAV was flown manually along various flight paths, such as those depicted in Figures 7c and 7d, in both the office of the 3D Geoinformation Research Group and Workshop 5 of the TU Delft Science Centre. Flightpaths depicted in Figures 7e for the corridor and 7f for the stairs were chosen, because the width of the corridor and stairs were about 2 meters and 1.5 meters respectively.

\begin{tabular}{|l|c|}
\hline Specifications & Parameters \\
\hline Sensor & CMOS $14 \mathrm{Mpx}$ \\
Optical & Sunny $180^{\circ}$ fish-eye $1 / 2.3^{\prime}$ aperture \\
Video stabilizer & lens: 3 -axis digital system \\
Video resolution & $1920 \times 1080 \mathrm{p}(30 \mathrm{fps})$ \\
Video encoding & $\mathrm{H} 264$ \\
\hline
\end{tabular}

Table 1. Specifications of the Parrot Bebop 2

The Parrot Bebop 2 (as seen in Table 1) is an ideal indoor aerial vehicle, since it is lightweight (about 500g) and can offer 25 minutes of continuous flight. Its high-performance specifications enable it to fly, film and take photographs indoors.Photoscan can generate high-quality $3 \mathrm{D}$ models based on video it captures. The use of the latest multi-view image capturing techniques without the need to set initial parameters (i.e. intrinsic camera parameters) is optimal when flying indoors. By eliminating the need for camera calibration, the use of the latest three-dimensional reconstruction technology allows for alignment of overlapping images needed for close-range photogrammetry. Image processing, can be conducting by using CIPs to improve the efficiency of alignment. Furthermore, our case studies suggest a wide range of factors should be considered when attempting indoor 3D reconstruction such as occlusion of CIPs by furniture, blank walls, glare from outdoors and other subtle factors negatively influencing alignment in Photoscan.

\subsection{Results}

We employed Photoscan to construct point clouds, as seen in Figures $10 a$ and $10 c$, and meshes, as seen in Figures 10b, 10d and $10 e$, of the office of the 3D Geoinformation Research Group in TU Delft, Workshop 5, and the outside stairs of the Workshop in the TU Delft Science Centre respectively. Our team found that noise in point clouds stem from four common sources: the glass in windows, blank walls, moving objects (people) and irregularlyshaped objects. The glass in windows is shiny and can be highly reflective or transparent. As such Photoscan matches points in the room to points outdoors, because it cannot differentiate between them. Moreover, in the case of white walls or uniformly-coloured surfaces, few features are detected and point cloud reconstruction 
is not properly performed. Therefore, they result in the generation of noisy point clouds. Other sources of noise that cannot be ignored are irregularly-shaped objects (based on the classification rules in Section 2) in indoor space (e.g. monitors, keyboards, fans, cables). Another possible cause of noise is the instability of the sensor itself, because the Photoscan has difficulty in calculating the internal orientation of the camera and therefore there may be problems aligning images that cause inappropriate tie points and noise in the point cloud. We also found that there are many holes in correspondence with floor point clouds, which were probably caused by an inability to locate CIPs on account of noise from uniform colours frequently caused by subjection to glare/reflectance. The presence of moving people during video recording was also found to cause noise in some cases.

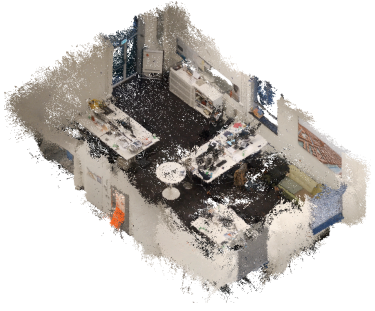

(a)

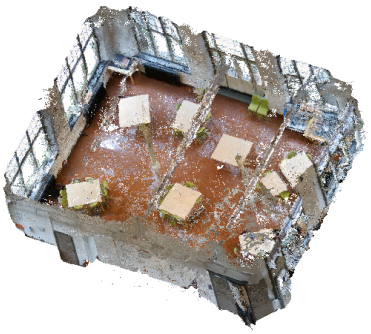

(c)

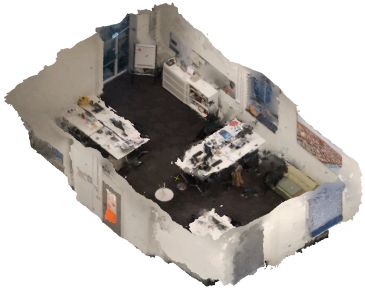

(b)

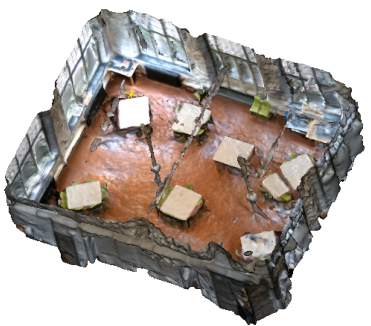

(d)

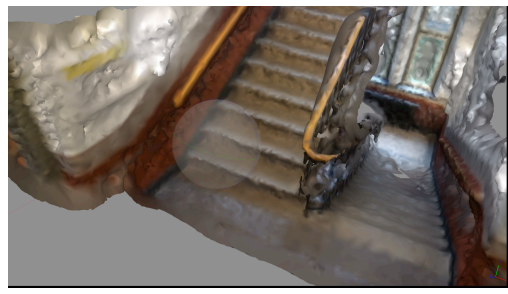

(e)

Figure 10. (a) and (b) are point cloud and mesh of the office of the 3D Geoinformation Research Group separately. (c) and (d) are point cloud and mesh of the TU Delft Science Centre Workshop 5. (e) is the mesh of stairwell.

\subsection{Discussion}

Flying QUAV for 3D indoor model reconstruction has many challenges; among them: safety issues, accessibility issues, object issues, semantic issues, light issues and noise issues. Semantic issues are related to reconstruction. Possibly we can address this issue by classifying objects based on a coloured point cloud, then assigning semantic information to them. Table 2 presents possible solutions to the challenges faced in aerial indoor video capture for $3 \mathrm{D}$ reconstruction purposes.

Furthermore, 3D models reconstructed by this approach have inherent advantages over free space (Diakité and Zlatanova, 2016) extraction, since they have free space only (see Figure 11), a shell model, see Figure $11 b$ and $11 d$, e.g. lockers, tables, etc. are concave space in the model. However, because of this inherent feature, models constructed by this method also have a big disadvantage that left out part of the space like the bottom of the table, where can be used as a escape space in emergency situations.

\begin{tabular}{|l|l|}
\hline Challenges & \multicolumn{1}{|c|}{ Potential solutions } \\
\hline Safety Issues & $\begin{array}{l}\text { Design flight paths based on safety dis- } \\
\text { tances, the FOV of the camera (Section } \\
\text { 3.3) and parameter estimation experi- } \\
\text { ments (Section 4.1). } \\
\text { Ask people to leave, negotiate with peo- } \\
\text { ple or fly the UAV during non-working } \\
\text { hours. } \\
\text { Remove, hide, or mask irregularly- } \\
\text { shaped objects along your flight path } \\
\text { whenever possible. Remove frames } \\
\text { Light Issues }\end{array}$ \\
$\begin{array}{l}\text { turnt on all lights in the modeled indoor } \\
\text { space, or conduct data acquisition on } \\
\text { sunny days. Remove frames with glare } \\
\text { prior to alignment } \\
\text { Put physical markers on blank walls, } \\
\text { single-colored floors, etc. Cover glass } \\
\text { portions of windows. Develop algo- } \\
\text { rithms to mask the glass on windows } \\
\text { automatically. Use UAV equipped with } \\
\text { excellent sensors. Use steady, patient } \\
\text { pilots to fly the UAV. }\end{array}$ \\
\hline
\end{tabular}

Table 2. Challenges in indoor UAV flight and potential solutions
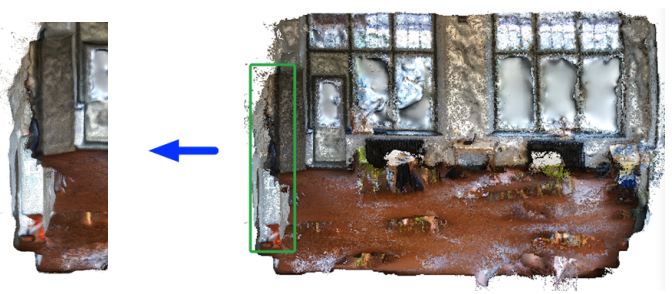

Figure 11. The area with green rectangle in the mesh model is a locker, which is a concave space from the bottom view.

\section{CONCLUSIONS}

In this paper, we investigated challenges of $3 \mathrm{D}$ indoor reconstruction based on video captured by flying a QUAV, the Parrot Bebop 2. Our results show that 3D models generated through indoor aerial video capture can be robust, especially with ultrahigh dense point cloud density in Agisoft Photoscan. Additionally, distances of roughly one meter between our QUAV's camera and objects targeted for 3D reconstruction was shown to be far superior to flight paths where video was captured at greater distances. Our results also indicate that attention should be paid to the shape of a chosen flight path, direction of the QUAV's camera and furniture which may occlude CIPs designated for image frame alignment in Photoscan. Moreover, it should be decided upon in advance which objects need to be reconstructed. If one's goal is to obtain an indoor 3D models with boundaries only, attention must only be paid to walls, floors and ceilings. If, however, realistic renderings of irregularly-shaped objects are desired, then 
close-range flight paths coupled with powerful image processing software is highly advantageous.

We intend to carry out future work to improve the quality of indoor 3D models from five standpoints:

1. Tests regarding density of markers on blank walls, singlecolored floors and furniture causing reflection, occlusion or noise. 2. Experiments to determine if there are techniques to aid alignment in Photoscan through savvy placement of markers at different heights, depths or in different patterns before recording the videos.

3. Research and/or development of algorithms to automate reliable CIPs placement in Photoscan.

4. Cultivation and testing of flight paths for QUAV in places where more than one previously tested flight path can be merged to create new flight paths to examine optimal routing configurations and camera directions in large indoor environments.

5. Take steps toward integration of autonomous QUAV flight capabilities through coordination with colleagues at TU Delft and other academic affiliates.

\section{ACKNOWLEDGEMENTS}

The financial support for this work comes from the program of China Scholarships Council (No. 201606410054). This work has been supported by the Dutch project STW/M4S 13742 'Smart 3D indoor models to support crisis management in large public buildings" (www.sims3d.net).

\section{REFERENCES}

Alsadik, B., Gerke, M. and Vosselman, G., 2015. Efficient use of video for $3 \mathrm{~d}$ modelling of cultural heritage objects. ISPRS Annals of the Photogrammetry, Remote Sensing and Spatial Information Sciences 2(3), pp. 1.

Boccardo, P., Chiabrando, F., Dutto, F., Tonolo, F. G. and Lingua, A., 2015. Uav deployment exercise for mapping purposes: Evaluation of emergency response applications. Sensors 15(7), pp. $15717-15737$.

Diakité, A. A. and Zlatanova, S., 2016. Extraction of the 3d free space from building models for indoor navigation. ISPRS Annals of Photogrammetry, Remote Sensing \& Spatial Information Sciences.

Gröger, G., Kolbe, T., Nagel, C. and Häfele, K., 2012. Opengis city geography markup language (citygml) encoding standard v2. 0.0. Open Geospatial Consortium Standard. Open Geospatial Consortium.

Hagedorn, B., Trapp, M., Glander, T. and Döllner, J., 2009. Towards an indoor level-of-detail model for route visualization. In: Mobile Data Management: Systems, Services and Middleware, 2009. MDM'09. Tenth International Conference on, IEEE, pp. 692-697.

Horna, S., Damiand, G., Meneveaux, D. and Bertrand, Y., 2007. Building $3 \mathrm{~d}$ indoor scenes topology from $2 \mathrm{~d}$ architectural plans. In: GRAPP $(G M / R)$, pp. 37-44.

Hornung, A., Wurm, K. M., Bennewitz, M., Stachniss, C. and Burgard, W., 2013. Octomap: An efficient probabilistic 3d mapping framework based on octrees. Autonomous Robots 34(3), pp. 189-206.

Koska, B., 2011. Determination of st. george basilica tower historical inclination from contemporary photograph. Geoinformatics FCE CTU 6, pp. 212-219.
Lee, J. and Zlatanova, S., 2008. A 3d data model and topological analyses for emergency response in urban areas. Geospatial information technology for emergency response. Taylor \& Francis Group, London pp. 143-165.

Lee, J., Li, K.-J., Zlatanova, S., Kolbe, T., Nagel, C. and Becker, T., 2014. Ogc indoorgml. Open Geospatial Consortium standard.

Li, K.-J., 2008. Indoor space: A new notion of space. In: International Symposium on Web and Wireless Geographical Information Systems, Springer, pp. 1-3.

Li, Y. and He, Z., 2008. 3d indoor navigation: a framework of combining bim with $3 \mathrm{~d}$ gis. In: 44th ISOCARP congress, pp. 110 .

LLC, G. M., 2009. Global mapper software llc. Parker, CO.

Manual, A. P. U., 2014. Professional edition. Aplastic Anemia (Hypoplastic Anemia).

Nex, F. and Remondino, F., 2014. Uav for 3d mapping applications: a review. Applied Geomatics 6(1), pp. 1-15.

Nikolov, I. and Madsen, C., 2016. Benchmarking close-range structure from motion 3d reconstruction software under varying capturing conditions. In: Euro-Mediterranean Conference, Springer, pp. 15-26.

Paasch, J. M., van Oosterom, P., Lemmen, C. and Paulsson, J., 2015. Further modelling of ladm's rights, restrictions and responsibilities (rrrs). Land use policy 49, pp. 680-689.

Remondino, F., Spera, M. G., Nocerino, E., Menna, F. and Nex, F., 2014. State of the art in high density image matching. The Photogrammetric Record 29(146), pp. 144-166.

Sebe, I. O., You, S. and Neumann, U., 2004. Dynamic objects modeling and 3d visualization. ASPRSO4, Colorado, May.

Stoffel, E.-P., Lorenz, B. and Ohlbach, H. J., 2007. Towards a semantic spatial model for pedestrian indoor navigation. In: $E R$ Workshops, Springer, pp. 328-337.

Strecha, C., 2011. Automated photogrammetric techniques on ultra-light uav imagery. Pix4D, http://www. pix $4 d$. com/downloads/pix4uav_accuracy. pdf pp. 1-11.

Teuliere, C., Eck, L., Marchand, E. and Guenard, N., 2010. 3d model-based tracking for uav position control. In: Intelligent Robots and Systems (IROS), 2010 IEEE/RSJ International Conference on, IEEE, pp. 1084-1089.

Yan, J., Shang, J., Yu, F., Zhiyong, Z. and Tang, X., 2016. Indoor spatial structure and mapping methods for real-time localization. Geomatics and Information Science of Wuhan University 41(8), pp. 1079-1086.

Yang, L. and Worboys, M., 2011a. A navigation ontology for outdoor-indoor space:(work-in-progress). In: Proceedings of the 3rd ACM SIGSPATIAL international workshop on indoor spatial awareness, ACM, pp. 31-34.

Yang, L. and Worboys, M., 2011b. Similarities and differences between outdoor and indoor space from the perspective of navigation. Poster presented at COSIT.

Zlatanova, S., Sithole, G., Nakagawa, M. and Zhu, Q., 2013. Problems in indoor mapping and modelling. Acquisition and Modelling of Indoor and Enclosed Environments 2013, Cape Town, South Africa, 11-13 December 2013, ISPRS Archives Volume XL-4/W4, 2013.

Revised July 2017 